\section{ON THE SPECTROSCOPE AND ITS APPLICATIONS VII.}

$A$ NOTHER point was also very obvious to those who $\mathrm{A}$ are familiar with these inquiries, namely, that if these prominences really consisted of gas, by the use of a powerful spectroscope it was perfectly unnecessary to wait for eclipses at all. The reason for this will be clear on a little consideration; if we take a continuous or unbroken spectrum and apply successively a number of prisms, the spectrum will become proportionately lengthened, and therefore more and more feeble, and in fact we can thus reduce the light to any degree required; if now, on the other hand, we take a spectrum which consists only of bright lines, say of one line in the red and another in the blue, and as before apply successively a number of prisms, we shall, it is true, increase the length of the spectrum, that is the distance between the two lines, but this will be all; the additional prisms have no power to alter the width of the lines themselves, for we have seen that these are simply the images of the slit, Their light, therefore, will only be slightly enfeebled. owing to reflection merely. Thus if we have a mixed light to analyse, part of which comes from a source giving out a continuous spectrum, and the rest that of a glowing gas, although when working with a single prism no lines may be visible on accoint of the brightness of the con-

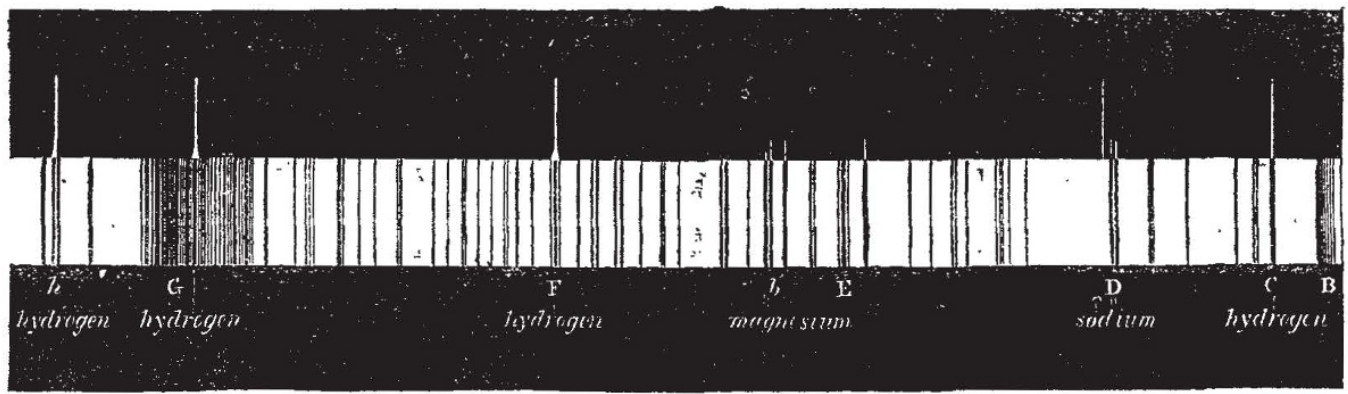

FIG. 40.--Spectrum of the Sun's Photosphere (below) and Chromosphere (above).

tinuous spectrum, yet by using say five or seven prisms we can so dilute the continuous spectrum as to render the bright lines of the glowing gas clearly visible. The case of the red flames round the sun is a case in point. They are invisible to the naked eye and in telescopes on account of the intensely illuminated atmosphere which also prevents anything like bright lines being observed from these red flames, until the bright continuous spectrum has been nuch reduced, when this has been done the bright lines of the spectrum, should there be any, will appear on a

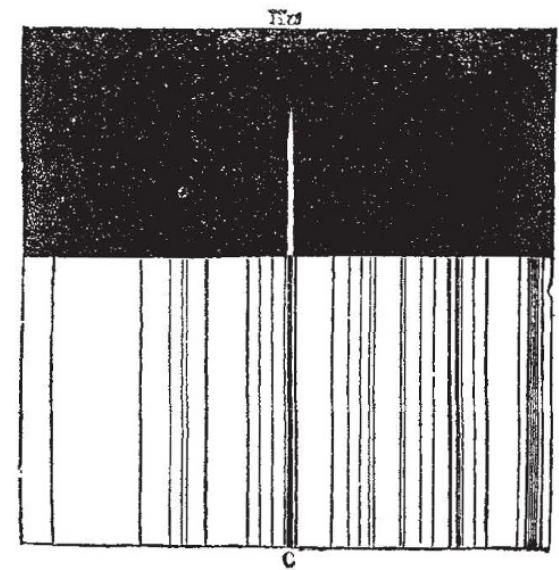

FIG. 4r.-C line bright in chromosphere, dark in sun.

comparatively dark background. M. Janssen, who was sent out by the French Government to observe the eclipse which was visible in India in 1868 , Major Tennant, and others, had no difficulty in recognizing in a moment, when the sun was eclipsed, that these things really did consist of gases or vapours, and $M$. Janssen, a very careful observer, had no difficulty in determining that the gas in question was really hydrogen gas. M. Janssen and myscif were also enabled to determine this by obser- vations on the uneclipsed sun, by means of the new method I have just sketched o:t. The accompanying woodc at (Fig. 40) shows the spectrum which is observed from these solar prominences. The spectrum of the prominences is shown in the upper, and that of the sun in the lower half of the engraving. This method is very easy to understand if you bear in mind the engraving of the spectroscope for solar work, and recollect that when we wish to examine the regions round the sun, the light of the sun is allowed to fall on the slit in such a way that

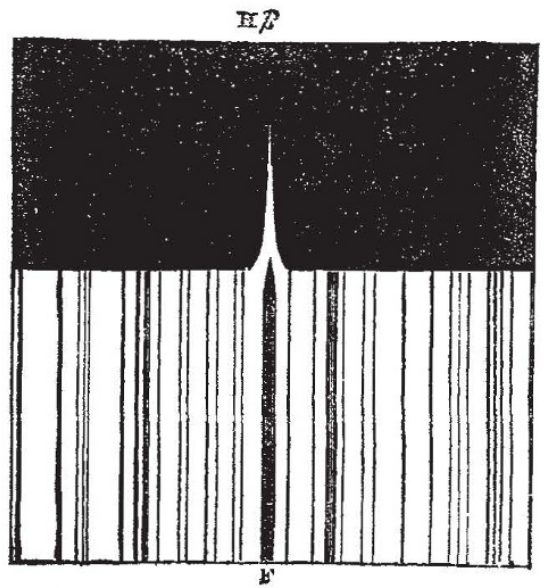

FIG. 42, $-F$ line in chromosphere, showing wideaing near the sun.

one half of the slit at the focus of the object glass of the large telescope is occupied by the brilliant image of the sun, and the other half is fishing, so to speak, around the limb or edge of the sun, so that if there is anything at all around the limb, the spectroscope, in the-to the eye-unoccupied part outside the image, picks up this something, and gives us its light sorted out into its proper bright lines in the spectrum. This spectrum shows that there is first a bright line, Fig. 4r, in 
the red, marked $\mathrm{C}$, which is absolutely coincident with a prominent dark line in the solar spectrum. Now this is a black line which, by repeated observations, we know corresponds in degree of refrangibility exactly with one of the lines given out by glowing hydrogen, when examined in one of these tubes with the electric spark. When, therefore, we get any substance around the sun reporting its light to us, it is perfectly obvious, I think, that if the bright line really be coincident with this dark line, that something is probably hydrogen. This was one of the first lines determined by $M$. Janssen in the eclipse of $\mathrm{r} 868$. There is another bright line absolutely coincident with a dark line known to correspond in refrangibility with another line given out by hydrogen in the green part of the spectrum, marked $F$ in the figure. This, then, is further proof in favour of hydrogen; and now notice a great difference between the shape of this line and the red line which I drew your attention to just now. An enlarged representation of this line is shown in Fig. 42.

You will bear in mind what I told you about the effect of pressure in altering the spectrum of hydrogen, and that one of the most obvious effects of increased pressure was to increase the thickness of what is called the $\mathrm{F}$ line-the line now under consideration, you will see here that the widening of the $\mathrm{F}$ line, the green line of hydrogen, really indicates a thickening due to pressure. In that way we have been able to determine approximately the pres-

DARK BAND IN MAGENTA.

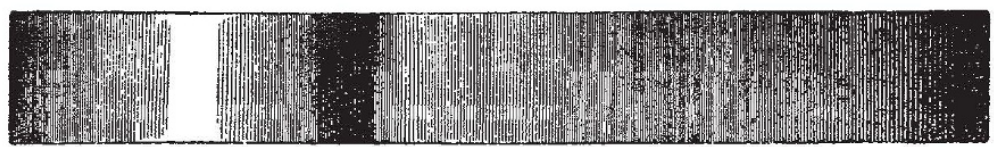

DARK BANDS IN BLOOD.

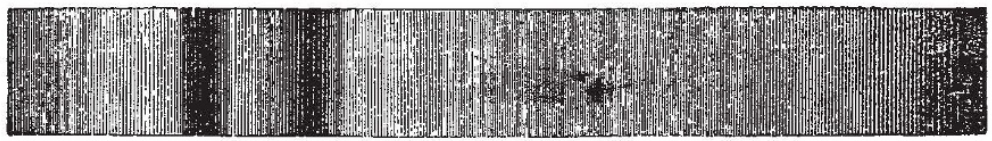

F1G. 43

sure of these circum-solar regions which the spectroscope has determined to be occupied by an envelope of hydrogen gas, mingled sometimes with other vapours, which envelope 1 have termed the chromosphere. When the pressure of the chromosphere is completely determined, we shall be probably enabled to determine the temperature of the sun.

A line again in the violet corresponds with a dark line in the solar spectrum, which is coincident with a third line of glowing hydrogen which we bave before spoken about, and there is still another coincident line. A line in the yellow of the spectrum wili also be noticed. This is one which has caused a great deal of discussion, for it is not coincident with any line of any known terrestrial substance. A number ot short lines are also shown in the engraving which will bseen to correspond to the part of the chromosphere which is denser, for then the $F$ line of hydrogen has becoms broad where these lines are seen; these lines show that in the layers of the chromosphere nearest to the sun a number of other substances exist, amongst which may be mentioned magnesium, iron, and sodium. The reason that bodies do not reach up so far from the body of the sun is that their vapours are very much heavier than the gas hydrogen, which is the lightest terrestrial substance known. Such are a few of the practical applications of the spectroscope as applied to the radiation of light. There are other classes of facts relating to the absorption of light, on the consideration of which we shall now enter.

The subject with which we have just been dealing is the radiation or giving out of light by bodies in different states - that is to say, by solid or liquid bodies, or gaseous or vaporous ones. We have now to deal with the action of the prism upon light under some new conditions-conditions which I purposely withheld from you in the last lecture. Light is not only given out, or radiated, but it may be stopped or absorbed in its passage from the light-source to our eye, if we interpose in the path of the beam certain more or less perfectly transparent substances, be they solids, liquids, gases, or vapours. I will recall one or two of the experiments which have been already described in order that you may see exactly how the perfectly distinct classes of phenomena due to radiation and absorption really run together. You will recollect that I pointed out to you that radiation, or the giving out of light, might be continuous or might be selective, and I am anxious now to show you that radiation is exactly equalled by absorption in this matter; that absorption may also be continuous or selective. We have before taken as an instance of continuous radiation a continuous spectrum obtained by using the electric lams or a lime-light; that is to say, an example of the general radiation which you get from an incandescent solid - the carbon points of which the poles

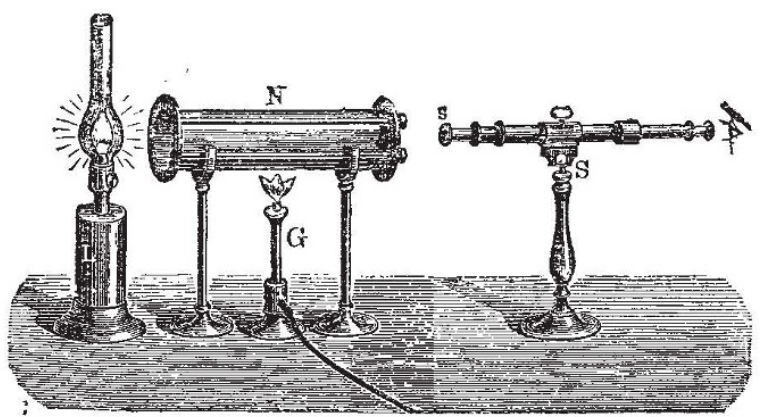

FIG. 44--Method of observing the absorption of a vapour

of the lamp are composed, or the solid lime. You will remember that if we take the spectrum of a vapour-as, for instance, that of strontium or thallium-we find that the continuous spectrum is altogether changed, and that in the place of that beautiful rainbow band, continuous from the red end of the spectrum to the violet, we really only get lines here and there, which are due to the selective radiation, and opposed to the general radiation which we spoke of in the continuous spectrum just now. I might have chosen other substances besides strontium, and thallium, but I mentioned the spectra of these substances when we were considering the question of radia- 
tion. What I have to dwell on now is, that the absorption or sifting of light by different bodies is very like radiation in its results - that is to say, in some cases we have an absorption which deals equally with every part of the spectrum, and in other cases we have absorption which only picks out a particular part of the spectrum here and there to act upon. But there is one important point to be borne in mind; when dealing with absorption we must always have a continuous spectrum to act upon. If we had a discontinuous spectrum to act upon, the thing would not be at all so clear. Having this continuous spectrum, the problem is, what the action of the different substances on the light will be. Let me give you an instance of general absorption. If we take the continuous spectrum above referred to, and interpose a piece of smoked glass, or better, a piece of neutral-tint glass, you will find that the substance will cut off the light and deaden the spectrum, so to speak, throughout its whole length. This neutral-tinted glass,

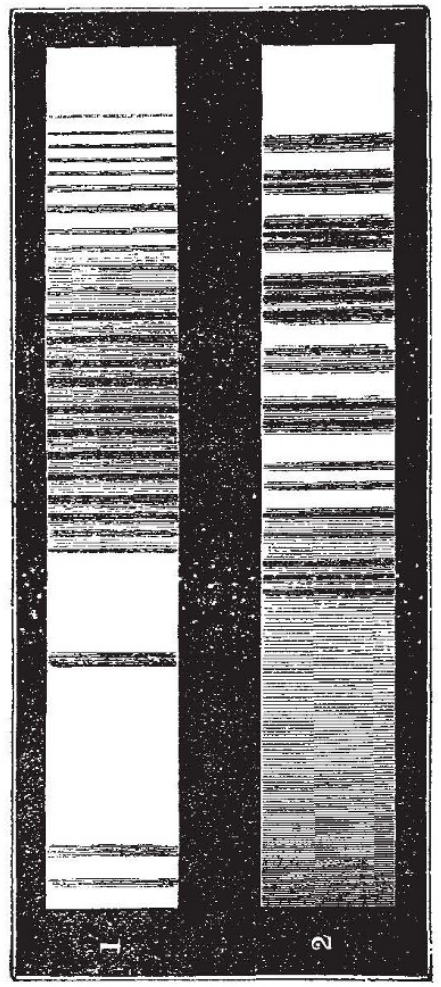

FIG 45,-Absorption spectra of iodine and nitrous fumes. violet. Now the fact that we can absorb any definite part of the spectrum by properly tinted glasses provides us with a practical application of spectrum analysis in the manufacture of the coloured glass used for lighthouses or signals. Further, if astronomers could find a glass of a certain red, or a glass of a certain green colour, we should be able to see the solar prominences every day without a spectroscope.

The first practical application which springs out of these phenomena of absorption is this, that as different substances are known by the effects which they produce on radiation, so also chemists find it perfectly easy to detect different substances by means of their absorption; for instance, the absorption spectrum of nitrous fumes can be shown by taking first our continuous spectrum, which we must always have to start with, and introducing some nitric peroxide between the source of light and the prism. The nitric oxide, immediately it comes in contact with the air, produces dense red fumes, and numbers of fine black lines will be seen immediately crossing the spectrum at right angles to its length, and to a certain extent resembling the solar spectrum with its Fraunhofer lines. Iodine is another substance which gives a coloured vapour, the absorption spectrum of which is very definite and well defined. Fig. 45, Spectrum No. 1, shows the absorption spectrum of iodine vapour, and No. 2 that of nitrous fumes. We are not limited to these substances; we will try something else-blood, for instance, about which I shall have something more to say presently. We shall find that the action of blood upon the light is perfectly distinct from the action of those fumes which we have spoken of; and instead of having typical lines in the green and blue parts of the spectrum, we have two very obvious lines in the more luminous part of the spectrum. The colour of a solution of blood is not unlike the colour of a solution of magenta ; but if, instead of using a solution of blood, we use a solution of magenta, we should have only a single black band. The absorption spectrum of potassic permanganate solution is another beautiful instance. We have here something totally unlike anything we had before. Instead of the two dark bands which we saw in the case of the blood, or the single band in the case of magenta, we have four very definite absorption bands in the green part of the spectrum. So that you see the means of research spectrum analysis affords as far as regards radiation, is entirely reproduced in the case of absorption, and it is perfectly easy, by means of the absorption of different vapours and d:fferent substances heid in solution, to determine not only what the absorbers really are, but to determine the presence of an extremely small quantity. Further, by allowing the light to pass through a greater thickness of the absorbing substance, the absorption lines are thickened and new regions of absorption are observed. This fact was discovered by Dr. Gladstone, who used hollow prisms containing the substance.

(To be continued.)

J. N. LOCKYER

\section{PROFESSOR ZOLLNER ON THE CONNECTION} BETWEEN COMETS AND METEORS

PROFESSOR F. ZÖLLNER alludes in the commencement of his paper read before the members of the "Kön. Sächs., Gesellschaft der Wissenschaften" to the epoch which Schiaparelli's discovery of the concordance of the orbits of some small comets with those of periodically returning showers of shooting stars has made in the astronomical world. He quotes an instance in proof of this, namely, Biela's Comet. On November 27 , last year, the earth was crossing the exact spot in her orbit, which had been cut by Biela's Comet two and a half months before. Observers aware of the coming event were on the alert with their instruments, but no good results were obtained owing to the unfavourableness of the weather.

From these facts, he says, we must naturally conclude that the physical constitution of these bodies is the same, and we are strengthened in our conclusions by Schiaparelli's discovery of the identity of the envelopes and tails of comets with clouds of meteors seen by reflected sunlight, the separate elements of which only become visible at a shorter distance.

Observations, however, with the spectroscope, contradict this assumption; the light given out by comets. is found not to correspond with that of the sun ; it is a light peculiar to them, like that of a glowing gas.

Further on he quotes Schiaparelli's own words to some length, with respect to the attraction exercised by other bodies on the matter composing the nuclei of comets, 